\title{
A DC-CIRCUIT MODEL OF THE PULSAR MAGNETOSPHERE
}

\author{
ShinPei Shibata \\ Department of Physics, Yamagata University
}

\begin{abstract}
The structure of the pulsar magnetosphere is studied in terms of a DC-circuit analogy. We find (1) the pulsar's death results from the 'disappearance' of operating points of this DC-circuit, (2) the outer gap is necessary, (3) the kinetic energy flux of the pair plasma wind and the electromagnetic flux are comparable, and (4) for rapid pulsars only two types of structure (a wind dominated one and an outer-gap dominated one) are possible.
\end{abstract}

\section{A global model}

The polar gap model accounting for the radio activity of pulsars (Ruderman and Sutherland 1975), the outer gap model for X-, $\gamma$-ray pulses from fast pulsars (Cheng, Ho, and Ruderman 1986a), and the thermal/centrifugal wind model for the pulsar wind (Michel 1969, Kennel, Fugimura, and Okamoto 1983), have been developed with great success. They all, however, are locally constructed; what is the global structure of the pulsar magnetosphere? Some parameters of the local models are fitted to what is observed; and the physical reasons for such parameters are not always clear. To solve these problems we search for possible global structure of the magnetosphere by using a simple DC-circuit model.

The simplest model of the pulsar magnetosphere would be a discharge tube connected to an electromotive force. We extend this model with the following characteristics of the local models in mind: (1) the outer gap is located just beyond the null surface and near the light cylinder, (2) the polar gap is well within the light cylinder and close to the stellar surface, (3) a strong electric current runs through both gaps, (4) pair plasmas are produced in the gap(s) to form the pulsar wind, and (5) centrifugal acceleration takes place in the wind where the current will be closed. Then we introduce a model with a closed current circuit as shown in figure 1.

Constructing local models with adjustable free parameters, we obtain characteristics, $V_{1}(I), V_{2}(I)$, $V_{\mathrm{W}}(I)$, which give the voltage drops of the polar and outer gaps and the pulsar wind, respectively, as functions of the current $I$ (different pairs of voltage and current correspond to different free parameters). We impose two global conditions to link the local models and obtain a global structure. The first one is Kirchhoff's second law $V_{0}=V_{1}+V_{2}+V_{W}$. Here $V_{0}$ is the electromotive force applied and given by $V_{0}=\Omega_{0} \Phi_{0} / 2 \pi c$, where $\Phi_{0}$ is the magnetic flux covering the circuit, and $\Omega_{0}$ is the angular veloc-

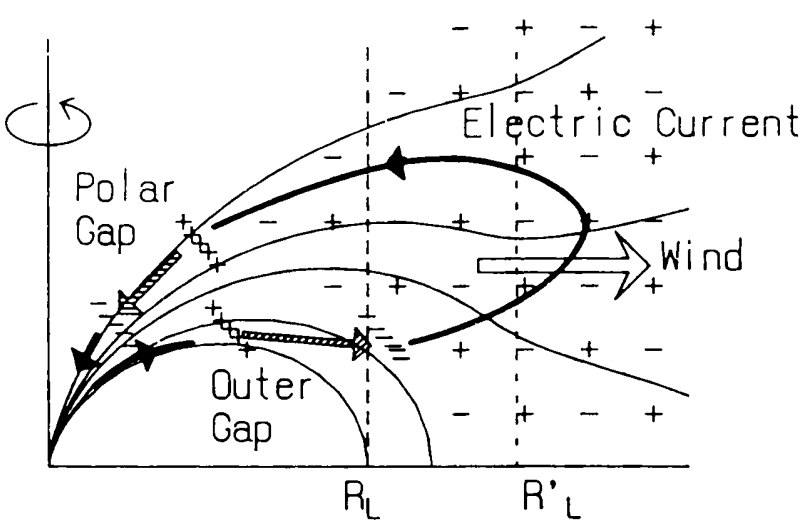

Figure 1a Schematic of the magnetospheric current circuit.

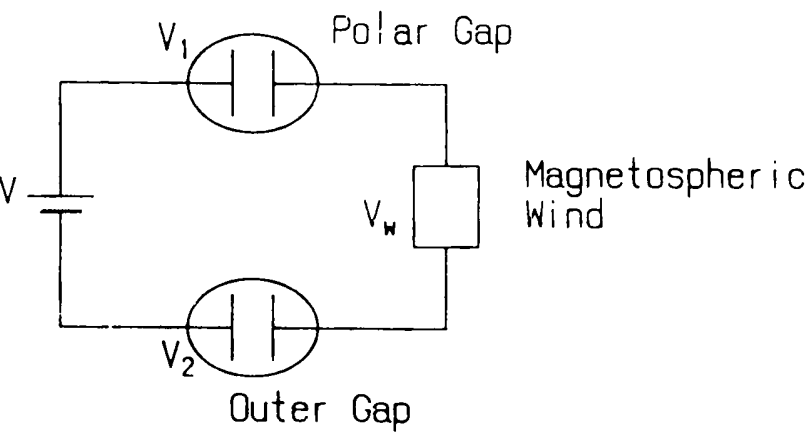

Figure 1b Equivalent circuit of the model. The structure of the magnetosphere can be studied in a similar way to the analysis of operating points of an electric circuit.

ity of the star. The outer magnetosphere rotates more slowly than the star because of the potential drop in the gaps $\left(\Omega<\Omega_{0}\right)$. As a result the pulsar wind with a larger torque $\operatorname{arm}$ of $R_{\mathrm{L}}^{\prime}=c / \Omega$ $\left(>R_{\mathrm{L}}=c / \Omega_{0}\right)$ conveys angular momentum efficiently. On the other hand, the polar gap emits little angular momentum. Thus the second global condition is the torque balance; i.e., if $\epsilon_{\text {total }}, \ell_{\text {total }}$ are loss rates of energy and angular momentum from the magnetosphere, $\epsilon_{\text {total }}=\Omega_{0} l_{\text {total }}$ should hold (we shall call this the 'energy-angular-momentum relation'). Using the local models with adjustable free parameters (characteristics of the circuit elements) and the two global conditions, we can obtain a global structure in a similar way to getting 
operating points of an electric circuit. After a brief description of the local models we will summarize the resulting global magnetospheric structure.

\section{The pulsar wind}

The acceleration power of the wind is $I V_{\mathrm{W}}$; which is added to the kinetic energy flow of the pair plasma. The pulsar wind also emits electromagnetic energy $\epsilon_{S}$ which is not used for the centrifugal acceleration. Therefore the total power of the wind is given by $\epsilon_{\mathrm{W}}=I V_{\mathrm{W}}+\epsilon_{S}$. The split monopole model suggests that the efficiency of the MHD acceleration $\eta=I V_{\mathrm{W}} / \epsilon_{S}$ is very small $\left(\eta \sim 10^{-4}\right.$ for the Crab), and the total current is much less than that expected by the gap current. This may indicate an incomplete wind theory; for an assumed non-radial field geometry we obtain a very different value of $\eta$ as a critical solution. In the present analysis we assume that the wind behaves so as to maintain the global conditions. Note that angular momentum loss by the wind is given by $\ell_{\mathrm{W}}=\epsilon_{\mathrm{W}} / \Omega$.

\section{The polar gap}

We calculate the space charge limited flow with the current density as a free parameter. The condition for pair creation gives the (current density vs. voltage) characteristic. Since we do not know how a cross section of the current in the polar gap is determined, we cannot help introducing a model parameter $\theta(0 \leq \theta \leq 1)$; if $\theta=1$, the whole polar cap is covered by the discharging current ${ }^{1}$. It must be noted that the polar gap $V_{1}$ has a lower limit because of the discharge condition. This causes the operating point to disappear, as discussed below.,

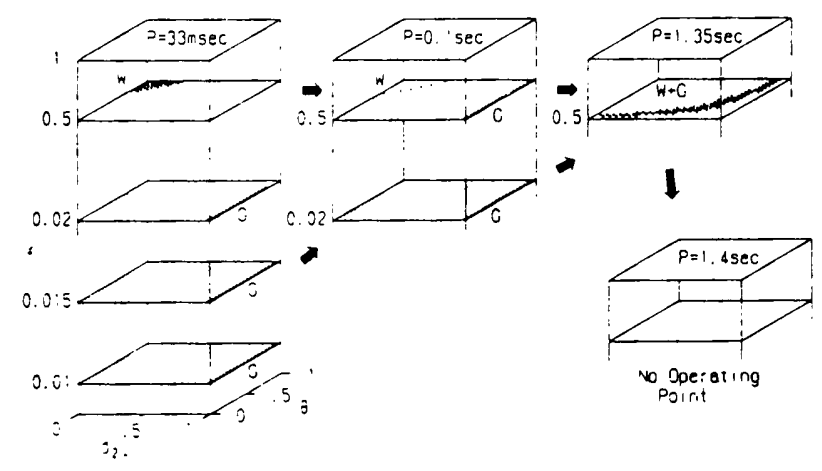

Figure 2 For a given period and magnetic momerit we have a three-dimensional parameter space $\left(f, \sigma_{2 \perp}, \theta\right)$ in which the dotted regions indicate that the operating points of the magnetospheric DC circuit exist. Only the thick dotted regions can be realistic pulsar models owing to restriction on the trans-field motion. ' $W$ ' and ' $G$ ' indicate that the corresponding structure is wind dominated and outer-gap dominated, respectively. The magnetic moment is assumed to be $2 \times 10^{30} \mathrm{G} \mathrm{cm}^{3}$.

${ }^{1}$ The effect of the inclination $\chi$ of the magnetic moment can be taken in $\theta$; for large $\chi, \theta$ becomes small.

\section{Outer gap}

The potential drop in the outer gap is composed of a trans-field component $V_{21}=\Omega_{0} \Psi_{G} / 2 \pi$ (where $\Psi_{G}$ is the magnetic flux in the outer gap) and a fieldaligned one $V_{2 \|}$. We introduce two non-dimensional parameters characterizing the outer gap; $f(\in 0,1)$ $V_{2 \|}$, and $\sigma_{2 \perp}(\in 0,1)$ measures the loss rate of angular momentum from the outer gap (mainly via hard photons) ${ }^{2}$.

\section{Results}

Figure 2 illustrates the results given below. The operating point disappears when the period is increased. This means death of the pulsar. Since the disappearance is due to the condition for pair creation (the lower bound of $V_{1}$ ), the physics for the death is essentially the same as that given by Sturrock (1971) and Ruderman and Sutherland (1975). It is found that the pulsar dies when $V_{1} / V_{0} \sim$ $0.1-0.2$. Note that the death condition is not given by $V_{1} / V_{0} \sim 1$. The reason is that the polar gap does not emit angular momentum efficiently; the outer magnetospheric activity should be kept at some level for the energy-angular-momentum relation to hold. The energy-angular-momentum relation leads us to two remarkable results: (1) the efficiency of the centrifugal acceleration should be very good. In other words, the kinetic energy flux of the wind is comparable to the Poynting flux, i.e., $\epsilon_{S} \sim I V_{\mathrm{W}}$, and (2) the magnetic flux in the wind should be less than the total flux of the circuit; as a result, the outer gap is necessary. For rapid pulsars $(P \lesssim 1 \mathrm{~s})$, only two types of magnetospheric structure are possible. One is the structure dominated by the pulsar wind, and the other is that dominated by the outer gap. For the Crab parameters, the windtype magnetosphere emits $99 \%$ of its power as the pulsar wind, about one half of which is carried by the plasma kinetic energy, and the remaining $1 \%$ is the power of the gaps, most of which is emitted as $\gamma$-ray and X-ray pulsed radiation. This result seems to agree with the observations. It should be noted here that the wind theory accounting for this strong wind is not known. The latter type (the outer-gap dominated one) corresponds to the circular flow model (Rylov 1979, Mestel, Phillips, and Wang 1979). For slow pulsars $(P \gtrsim 1 \mathrm{~s})$, the power of the wind and outer gap are comparable and the two types are merged.

\footnotetext{
${ }^{2} f$ denotes the fraction of $V_{1}$ to the total field aligned voltage gap, i.e., $V_{1}=f V_{\|}, V_{2 \|}=(1-f) V_{\|} . \sigma_{21}$ is so defined that the angular momentum loss from the outer gap is $I V_{2} \sigma_{2} \perp / \Omega_{0}$.
} 